\title{
World Workshop on Oral Medicine VII: Prognostic biomarkers in oral leukoplakia: A systematic review of longitudinal studies
}

\author{
Alessandro Villa ${ }^{1,2^{*}}$ (1) | Antonio Celentano ${ }^{3^{*}} \mid$ Ingrid Glurich $^{4}$ | Wenche S. Borgnakke ${ }^{5}$ | \\ Siri Beier Jensen $^{6}$ | Douglas E. Peterson ${ }^{7}$ (D) | Konstantina Delli ${ }^{8}$ (i) | David Ojeda ${ }^{9}$ | \\ Arjan Vissink ${ }^{8}$ (D) Camile S. Farah ${ }^{10}$ \\ ${ }^{1}$ Division of Oral Medicine and Dentistry, Brigham and Women's Hospital, Boston, Massachusetts \\ ${ }^{2}$ Department of Oral Medicine, Infection and Immunity, Harvard School of Dental Medicine, Boston, Massachusetts \\ ${ }^{3}$ Melbourne Dental School, The University of Melbourne, Melbourne, Victoria, Australia \\ ${ }^{4}$ Center for Oral and Systemic Health, Marshfield Clinic Research Institute, Marshfield, Wisconsin \\ ${ }^{5}$ Department of Periodontics and Oral Medicine, School of Dentistry, University of Michigan, Ann Arbor, Michigan \\ ${ }^{6}$ Department of Dentistry and Oral Health, Faculty of Health, Aarhus University, Aarhus, Denmark \\ ${ }^{7}$ Oral Medicine Section, Department of Oral Health and Diagnostic Sciences, School of Dental Medicine, UConn Health, Farmington, Connecticut \\ ${ }^{8}$ Department of Oral and Maxillofacial Surgery, University of Groningen, University Medical Center Groningen, Groningen, The Netherlands \\ ${ }^{9}$ Department of Comprehensive Dentistry, School of Dentistry, UT Health San Antonio, San Antonio, Texas \\ ${ }^{10}$ Australian Centre for Oral Oncology Research \& Education, Perth, Westren Australia, Australia
}

\section{Correspondence}

Alessandro Villa, Division of Oral Medicine and Dentistry, Brigham and Women's

Hospital, Boston, MA.

Email: avilla@bwh.harvard.edu

\section{Funding information}

American Academy of Oral Medicine;

European Association of Oral Medicine;

The British Society for Oral Medicine; The

National Institute of Dental and Craniofacial

Research; Colgate-Palmolive; Henry

Schein Cares Foundation; AFYX; Unilever;

Xerostom; Oral Diseases; The World Dental

Education Foundation

\begin{abstract}
Objective: To identify the prognostic biomarker candidates for stratification and long-term surveillance of oral leukoplakia progressing to cancer via a systematic literature review.

Materials and Methods: Systematic searches with no date restrictions were conducted on March 29, 2018, targeting the databases PubMed (Ovid), EMBASE (Ovid), EBM (Ovid), and Web of Science (ISI). Bias was assessed using the Quality in Prognosis Studies tool. Biomarkers were stratified based on hallmarks of cancer.

Results: Inclusion criteria were met by 25 of 3,415 studies. A range of biomarkers were evaluated experimentally for risk stratification, prognosis, and surveillance of oral leukoplakia in tissue, blood, and saliva. However, the studies were highly heterogeneous and require further validation. Biomarkers reported in these studies included inflammatory or oxidative markers, growth factors, ion channels, genetic and cellular regulatory factors, and epigenetic biomarkers. Studies tended to include small sample sizes, under-reported or variably reported histopathological data, did not address potential confounding, reported limited/variable follow-up data, or lacked a control group. Inclusion of subsets from chemoprevention trials may have introduced bias regarding reported malignant transformation rates and accuracy of prognostic biomarkers.
\end{abstract}


Conclusions: This review identified insufficient longitudinal evidence to support validated prognostic biomarkers for oral leukoplakia. Further studies are needed to identify molecular targets with the potential to mitigate risk of malignant transformation.

\section{KEYWORDS}

biomarkers, cancer progression, leukoplakia, neoplastic cell transformation, prognosis, tumor

\section{1 | INTRODUCTION}

Oral squamous cell carcinoma (OSCC) continues to have significant clinical and economic impact at the international level. It is associated with a high rate of mortality, which often is a consequence of delayed diagnosis due to lack of screening programs, low health literacy relative to OSCC, and lack of access to care. The World Health Organization (WHO) projected 354,864 new cases of oral cavity and lip cancer in 2018 (Bray et al., 2018).

Oral potentially malignant disorders (OPMDs), of which leukoplakia is one of the several phenotypes, represent a group of conditions and lesions with variable propensity for oncogenic potential (Warnakulasuriya, Johnson, \& van der Waal, 2007). In the era of precision medicine, there is burgeoning interest in defining and characterizing relative risk of oral cancer emergence in association with OPMD. As the understanding of the molecular pathogenesis of oral cancer continues to expand, there is active interest in identifying biomarkers that could provide ability for clinicians to longitudinally track key molecular signals associated with OPMD, and intervene prior to neoplastic transformation. The overarching aims of the systematic reviews performed by the Precision Medicine Group of World Workshop on Oral Medicine VII were to:

- Assess whether prognostic biomarkers could accurately stratify the risk of malignant transformation of oral leukoplakia.

- Assess the relationship between prognostic biomarkers and the patient's risk profile including lesion clinicopathologic characteristics in addition to patient's risk factors.

- Evaluate whether biomarkers could independently predict malignant transformation of oral leukoplakia.

- Establish the minimum follow-up intervals required for biomarkers to predict malignant transformation.

- Assess the efficacy of the investigated biomarkers and management protocol.

- Formulate an algorithm that would help clinicians to provide the best supported evidence-based management protocol to patients with oral leukoplakia.

After oral submucous fibrosis, oral leukoplakia is the most common OPMD, with a worldwide prevalence of $4.11 \%(95 \% \mathrm{Cl}$ : 1.98-6.97) (Mello et al., 2018). Leukoplakia has been defined by the $\mathrm{WHO}$ as "a white plaque of questionable risk having excluded (other) known diseases or disorders that carry no increased risk for cancer" (Warnakulasuriya et al., 2007). Risk factors for oral leukoplakia are similar to those observed in oral cancer and include tobacco smoking, heavy alcohol consumption, areca nut chewing (especially in South Asian countries), immunosuppression (e.g., HIV/AIDS, post-organ transplantation), personal or family history of cancer (60\%-70\%), ultraviolet light exposure (for lip lesions only), and selected syndromes (e.g., dyskeratosis congenita) (Villa \& Woo, 2017; Warnakulasuriya, 2018).

Leukoplakia is a clinical diagnosis, most commonly presenting in two main phenotypes: homogeneous and non-homogeneous leukoplakia. Proliferative verrucous leukoplakia represents a third, rarer, high-risk subtype (Warnakulasuriya, 2018). Irrespective of type of oral leukoplakia, the gold standard for final diagnosis remains incisional biopsy. Risk of malignant transformation depends on the clinical form and the grade of dysplasia, although other clinical and histopathological parameters have been reported as drivers (Speight, Khurram, \& Kujan, 2018). Non-homogeneous leukoplakias carry a $20 \%-25 \%$ risk of cancer progression versus $0.6 \%-5 \%$ in homogeneous cases (Napier \& Speight, 2008; Reibel, 2003; van der Waal \& Axell, 2002).

A key step to better understanding oral leukoplakia outcomes is to identify the molecular factors that drive malignant progression, as these factors may also represent attractive candidates for targeted therapies. With the advent of precision medicine, a growing evidence base has explored predictive and prognostic biomarkers for oral leukoplakia. This paper systematically reviewed longitudinal studies which specifically aimed to: (a) assess whether prognostic biomarkers could accurately stratify the risk of progression of oral leukoplakia to cancer, and (b) evaluate the reliability of biomarkers in long-term surveillance of oral leukoplakia. Future studies will focus on the other overarching aims as mentioned above.

\section{2 | MATERIALS AND METHODS}

This study was conducted by the Precision Medicine Work Group within the World Workshop on Oral Medicine VII (WWOM VII). Results are reported according to the Preferred Reporting Items for Systematic Reviews and Meta-Analyses (PRISMA) statement (Moher, Liberati, Tetzlaff, \& Altman, 2009). PICO (Patients, Intervention/ exposure/prognostic factor, Comparison group and Outcome) was used to formulate the research question.

- Patients: patients with oral leukoplakia

- Intervention/exposure/prognostic factor: biomarkers in human specimens (saliva, blood, gingival crevicular fluid, oral tissues) 
$29^{\text {th }}$ March 2018

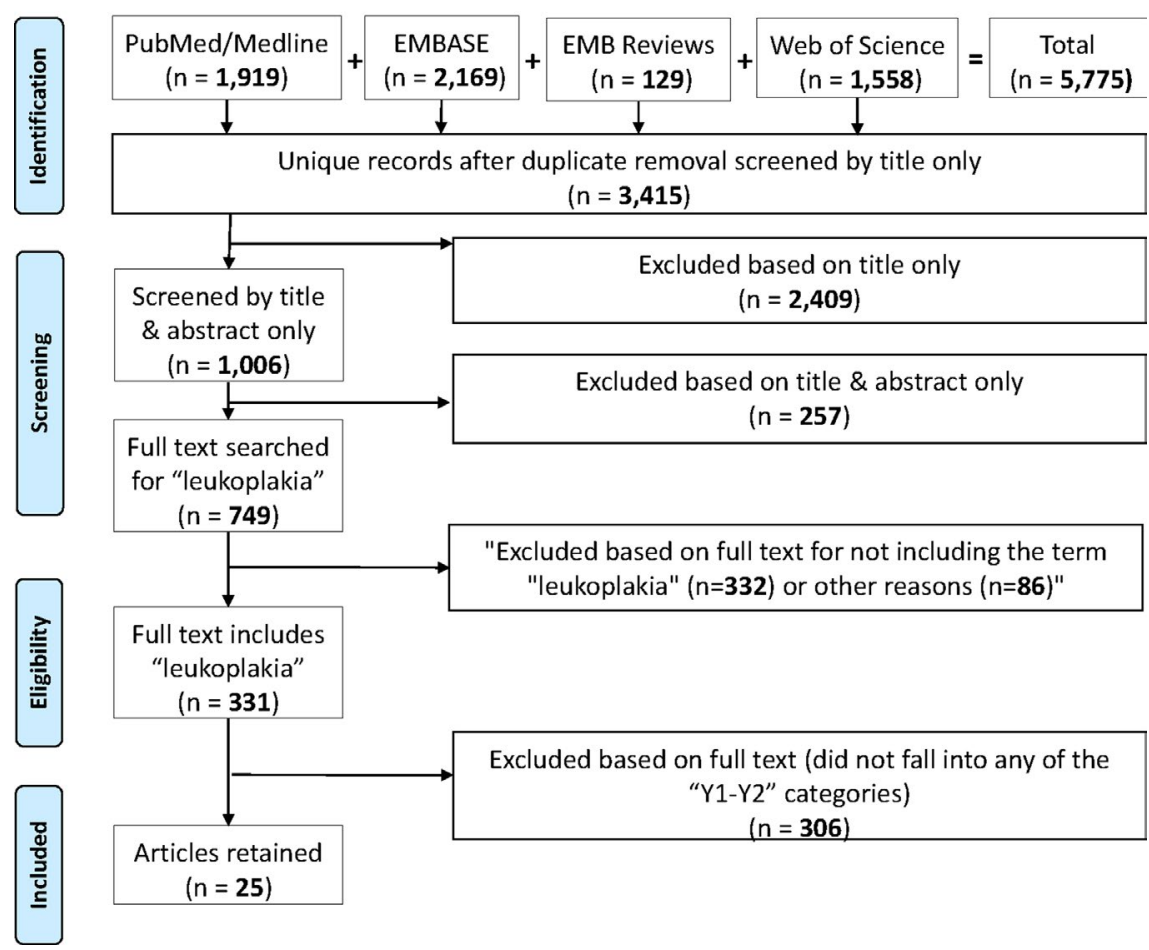

FIGURE 1 Selection of studies for systematic review of prognostic biomarkers for oral leukoplakia
- Comparison: healthy controls or patients with oral squamous cell carcinoma

- Outcome: squamous cell carcinoma

- Studies: longitudinal (prospective) studies

\section{1 | Study selection}

Studies that were potentially eligible for inclusion evaluated biomarker expression in: (a) human specimens (saliva, blood, gingival crevicular fluid, oral tissues) with follow-up data (over time); (b) patients with oral leukoplakia compared to healthy controls; or (c) patients with oral leukoplakia compared to patients with oral squamous cell carcinoma. Studies were excluded if they were: (a) studies investigating only non-human tissues, (b) withdrawn/ retracted studies, (c) reviews, (d) case reports, (e) commentaries, (f) opinion articles, $(\mathrm{g})$ letters to the Editor, or ( $h$ ) congress abstracts.

The selection of studies for review was conducted in the following five steps as illustrated in Figure 1.

Step 1: Electronic literature searches were conducted on March 29, 2018, using the PubMed (Ovid), EMBASE (Ovid), Evidence Based Medicine (EBM) Reviews (Ovid), and Web of Science (ISI) databases with no publication year restrictions. The search strategies according to the syntax rules of each database are displayed in Supporting Information Table S1. The identified citations were imported into the electronic database EndNote X8 (Clarivate Analytics, Philadelphia, PA, USA). Deduplication was achieved by the EndNote software and manually by the two reviewers (AVil and $A C$ ).
Each subsequent step was conducted by two blinded reviewers (AVil and AC) to exclude records ineligible for inclusion, based on sequential review of title only, title and abstract, and finally full text (Step 4). The applied exclusion categories are shown in Table 1. When more than one exclusion category would pertain to a study, the most important was selected. At each step, review discordance between the reviewers was resolved by discussion between the two reviewers (AVil and $A C$ ) under supervision of the senior reviewer (CSF).

Step 2: Among unique records screened by title, only 1,006 out of 3,145 were retained. Cohen's kappa statistic for inter-reviewer agreement was 0.95 ( $95 \% \mathrm{Cl}: 0.94-0.96)$, and the absolute agreement between the two reviewers was $96.7 \%$.

Step 3: Screening by both title and abstract resulted in retention of 749 of 1,006 for full-text review. The absolute agreement between the two reviewers was $96.7 \%$, and the kappa statistic was 0.92 (95\% Cl: 0.91-0.94).

Step 4: Based on review of the full text, 331 of 749 were retained based on identification of "leukoplakia" as the definitive diagnosis. The reasons for excluding the 418 studies are shown in Table 1.

Step 5: The remaining 331 papers were allocated to one or more of the following eligibility categories, which respectively assessed the efficacy of biomarkers in:

- Y1: stratifying the risk of progression of oral leukoplakia to cancer (prospective longitudinal studies only).

- Y2: long-term surveillance of oral leukoplakia (longitudinal studies only). 
TABLE 1 Reason for exclusion after full-text assessment

\begin{tabular}{|l|l|l|}
\hline Exclusion category & Reason for exclusion & Number of studies \\
\hline N0 & Not English language & 17 \\
\hline N1 & $\begin{array}{l}\text { Not original study (review, guideline, } \\
\text { editorial, conference abstract) }\end{array}$ & 2 \\
\hline N2 & $\begin{array}{l}\text { No human tissue or only immortalized } \\
\text { human cell lines in vitro }\end{array}$ & 3 \\
\hline N3 & $\begin{array}{l}\text { No clinical diagnosis of oral leukoplakia, only } \\
\text { dysplasia }\end{array}$ & 332 \\
\hline N4 & $\begin{array}{l}\text { Cancer patients only: resection margins/ } \\
\text { perilesional tissue in oral/oropharynx } \\
\text { squamous cell carcinoma; history of cancer } \\
\text { prior to or during study; undergoing } \\
\text { chemotherapy or radiation therapy }\end{array}$ & 6 \\
\hline N5 & $\begin{array}{l}\text { Predatory journals } \\
\text { Retracted articles, or title includes } \\
\text { "expression of concern" }\end{array}$ & 2 \\
\hline N7 & $\begin{array}{l}\text { Other reason for not meeting inclusion } \\
\text { criteria }\end{array}$ & 53 \\
\hline All (N1-N7) & \begin{tabular}{l} 
Reports ineligible for inclusion \\
\hline
\end{tabular} & 418 \\
\hline
\end{tabular}

- Y3: diagnosis of oral leukoplakia as an adjunct to oral examination (prospective case-control/cross-sectional studies only)

- Y4: progression of oral leukoplakia in a retrospective data set (case-control/cross-sectional studies only)

- Y5: differentiating oral leukoplakia from controls; correlation of differential biomarker expression with diverse clinicopathologic parameters (case-control/cross-sectional studies only).

Inter-reviewer absolute agreement for Step 5 was $85.47 \%$, and the kappa statistic was 0.59 (95\% Cl: $0.50-0.68)$, but $100 \%$ absolute agreement was reached upon a second revision.

As per title, the current report was limited to the 25 longitudinal studies included in the categories $Y 1$ regarding progression risk and Y2 on long-term surveillance. Consequently, 306 of 331 studies classified by $Y$ categories that did not meet eligibility for inclusion in $\mathrm{Y} 1$ or $\mathrm{Y} 2$ categories either due to lack of eligibility for any of the $Y$ category or because they were included into categories $\mathrm{Y} 3-\mathrm{Y} 5$ were excluded from further review in the current report. Of note, all papers included in the $\mathrm{Y} 2$ category were also included in the Y1 category and the results will therefore be reported collectively.

The reviewer (AVil) and the consultant (CSF) extracted and entered into specifically developed forms (Microsoft Excel 2010, Redmond, Washington, USA) all relevant data from the selected papers. Risk of bias for the 25 included studies was assessed independently by one reviewer (AVil) and the consultant (CSF) using the "Quality in Prognosis Studies" (QUIPS) tool (Hayden, van der Windt, Cartwright, Cote, \& Bombardier, 2013), which evaluates the six domains "study participation," "study attrition," "prognostic factor measurement," "outcome measurement," "study confounding," and "statistical analysis and reporting." Discord was resolved by consensus.

\section{2 | Statistical analysis}

Absolute percent inter-reviewer agreement and Cohen's kappa coefficient were calculated using IBM Statistics 23 (SPSS, Chicago, Illinois, USA). The heterogeneity of the studies and the high number of different biomarkers studied prevented any quantitative analysis of the results, so no meta-analysis was performed.

\section{3 | RESULTS}

Of the 331 publications eligible for the categories Y1-Y2 out of the originally identified 3,415 unique records, 25 studies were allocated to groups $\mathrm{Y} 1$ and $\mathrm{Y} 2$ and therefore included in this report (Figure 1). Results are reported by types of biomarkers stratified to the hallmarks of cancer (Hanahan \& Weinberg, 2011). The main characteristics of the studies are presented in Table 2, and a detailed description of the biomarkers identified is reported in the Supporting Information Appendix S1.

\section{1 | Types of biomarkers}

Biomarkers reported in these studies (Table 2) included inflammatory or oxidative markers (Chang et al., 2013; Massarelli et al., 2005; Rai, Kaur, Jacobs, \& Singh, 2010), growth factors (Beenken et al., 1994, 1999; Uehara, Ikeda, Nonaka, \& Asahina, 2010; Wan, Meyskens, Armstrong, Taylor, \& Kennedy, 1999), cell signaling biomarkers (Saintigny et al., 2011, 2018, 2018; Sakata et al., 2017), genetic and cellular regulatory factors (Lee et al., 2000; Mao et al., 1996; Nagao et al., 2017; Tanic, Tanic, Milasin, Vukadinovic, \& Dimitrijevic, 2009; Visioli, Lauxen, Sant'ana Filho, \& Rados, 2012), 


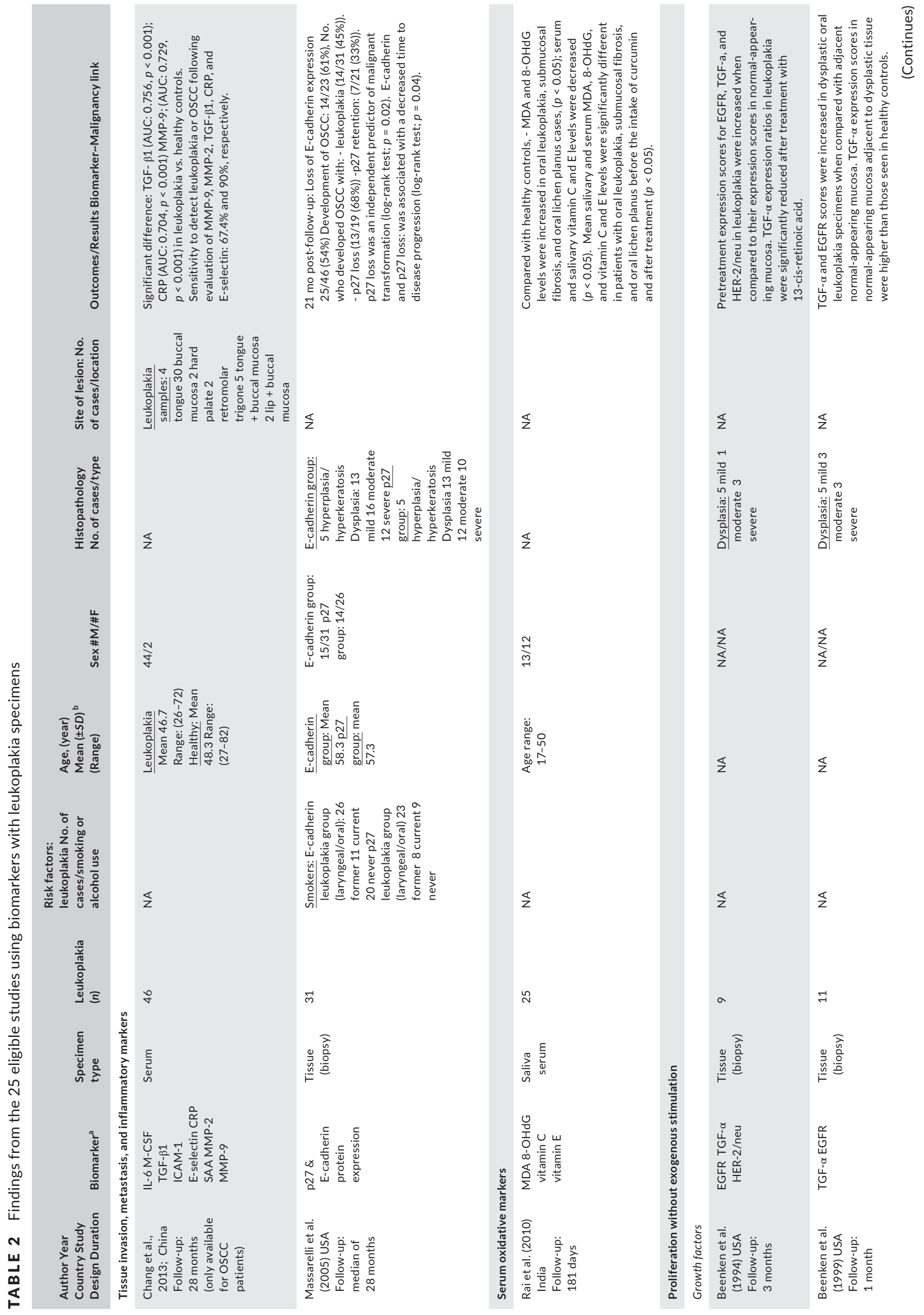



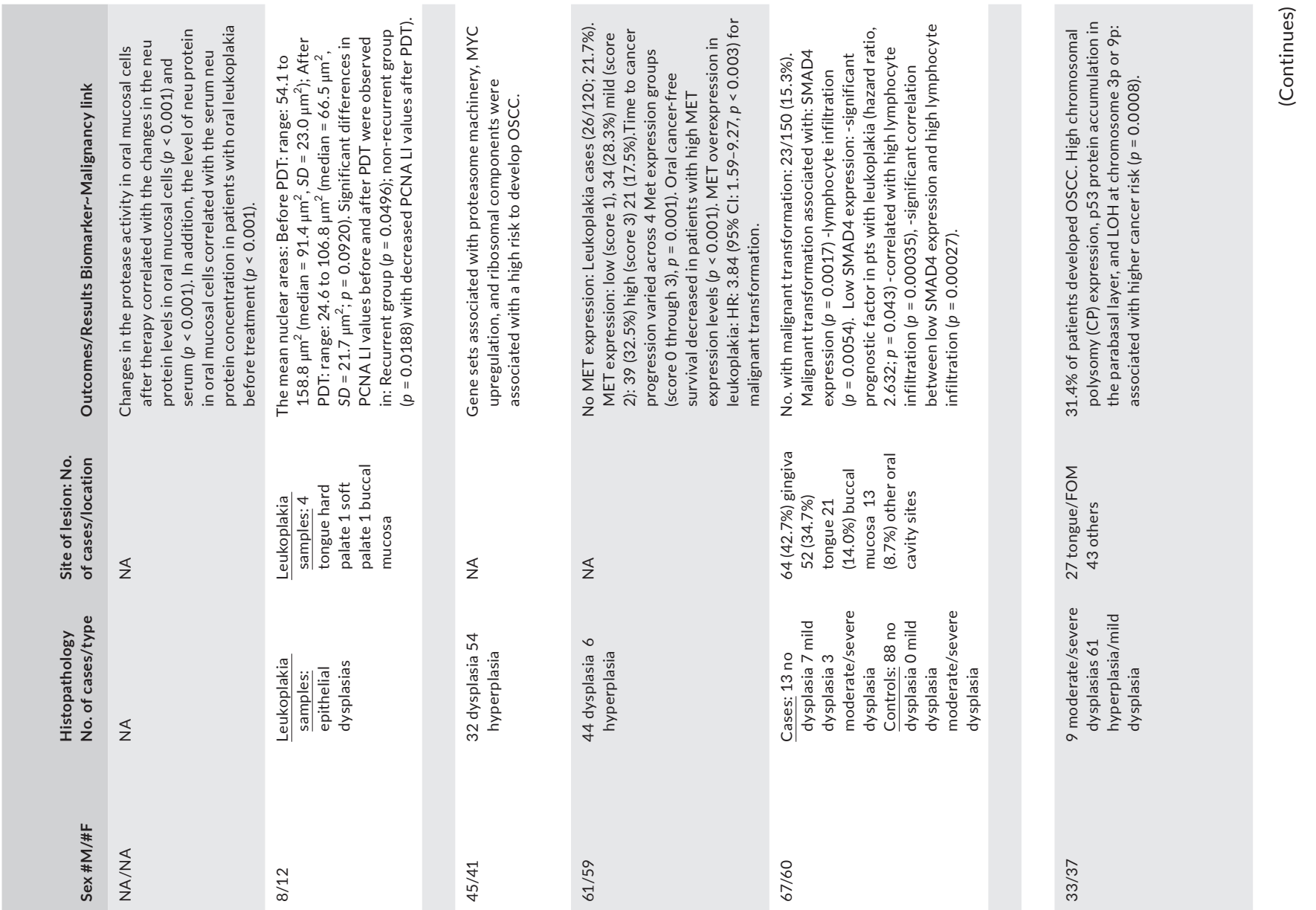

$\frac{1}{8}$

誉
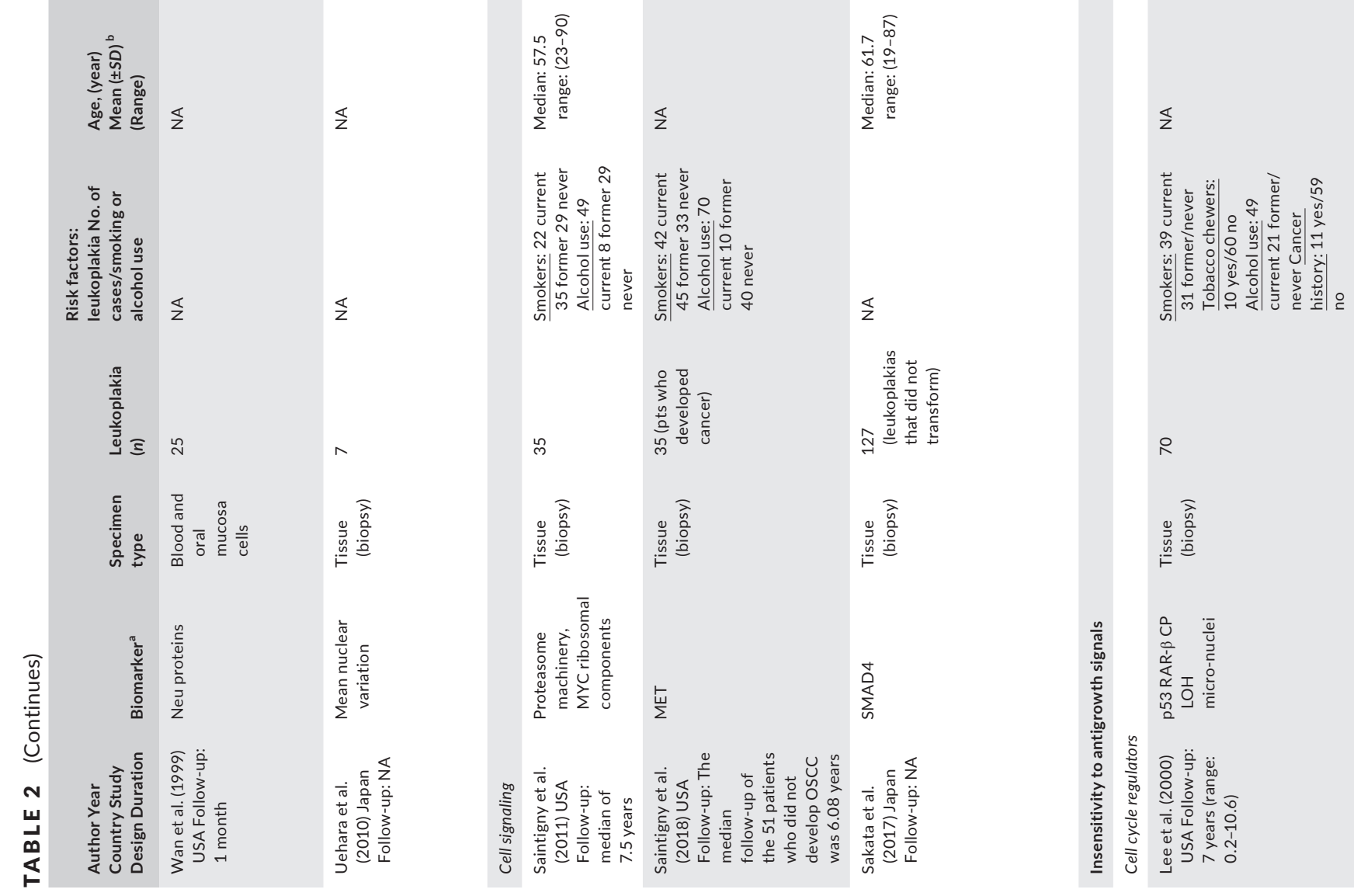


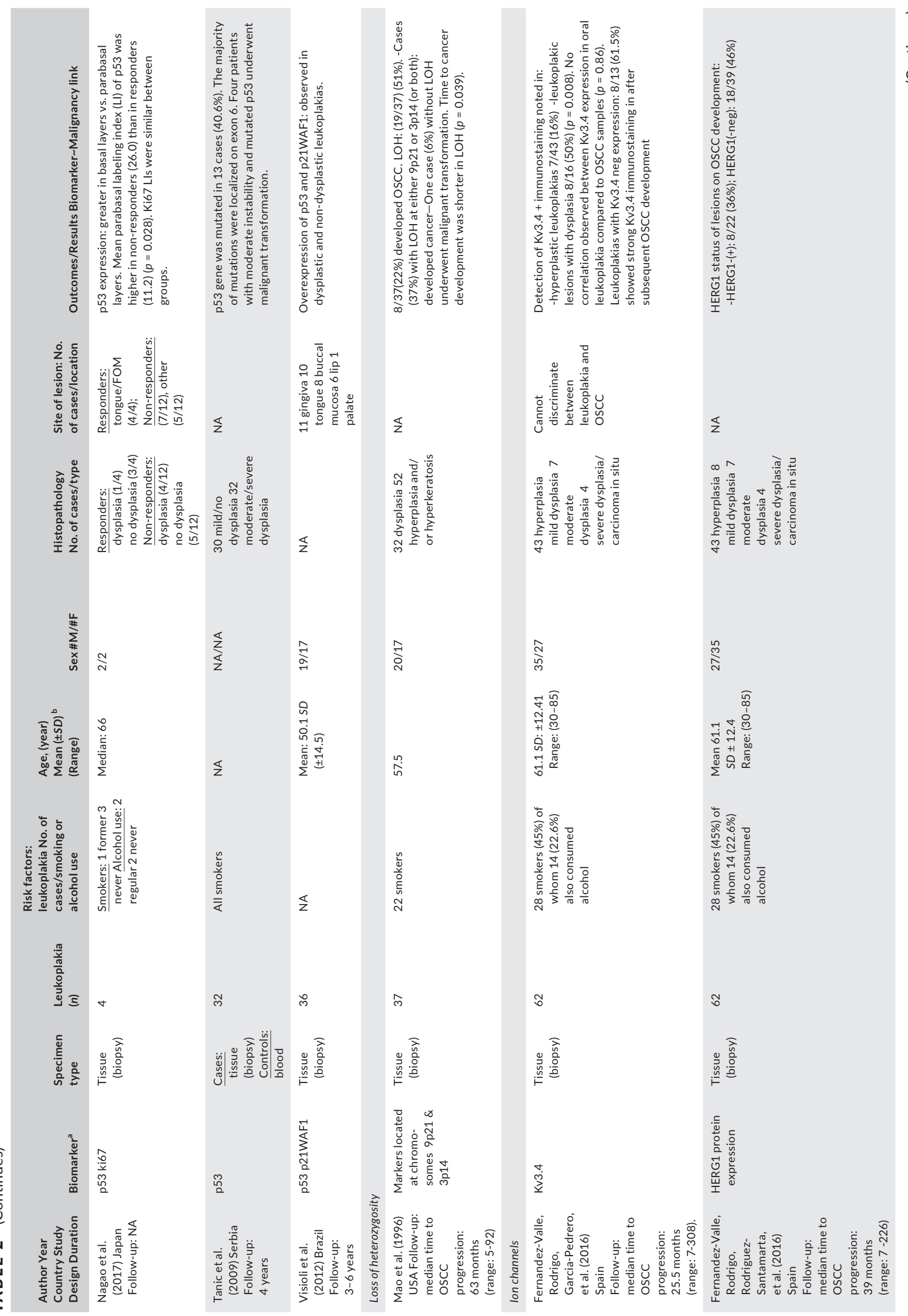




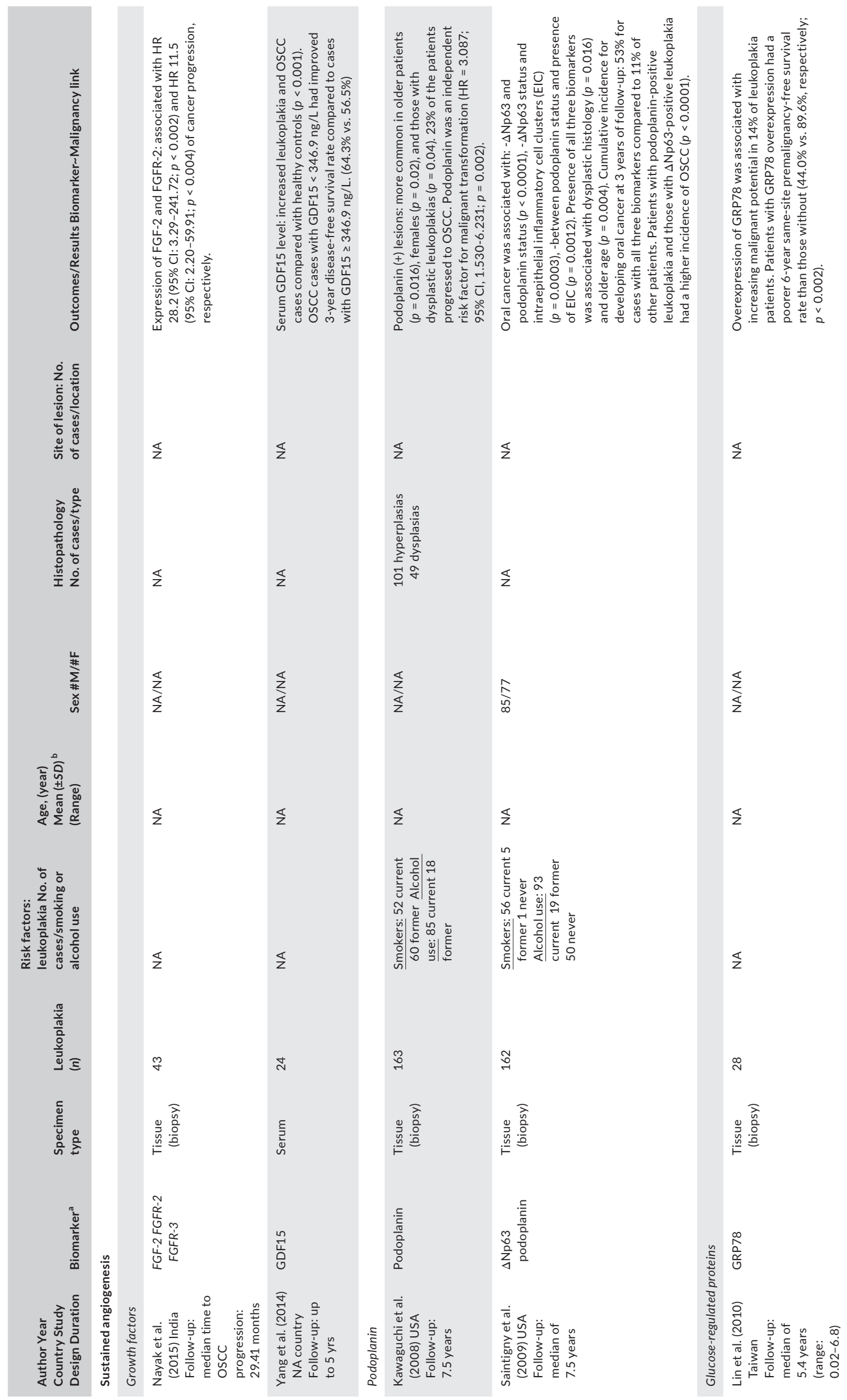




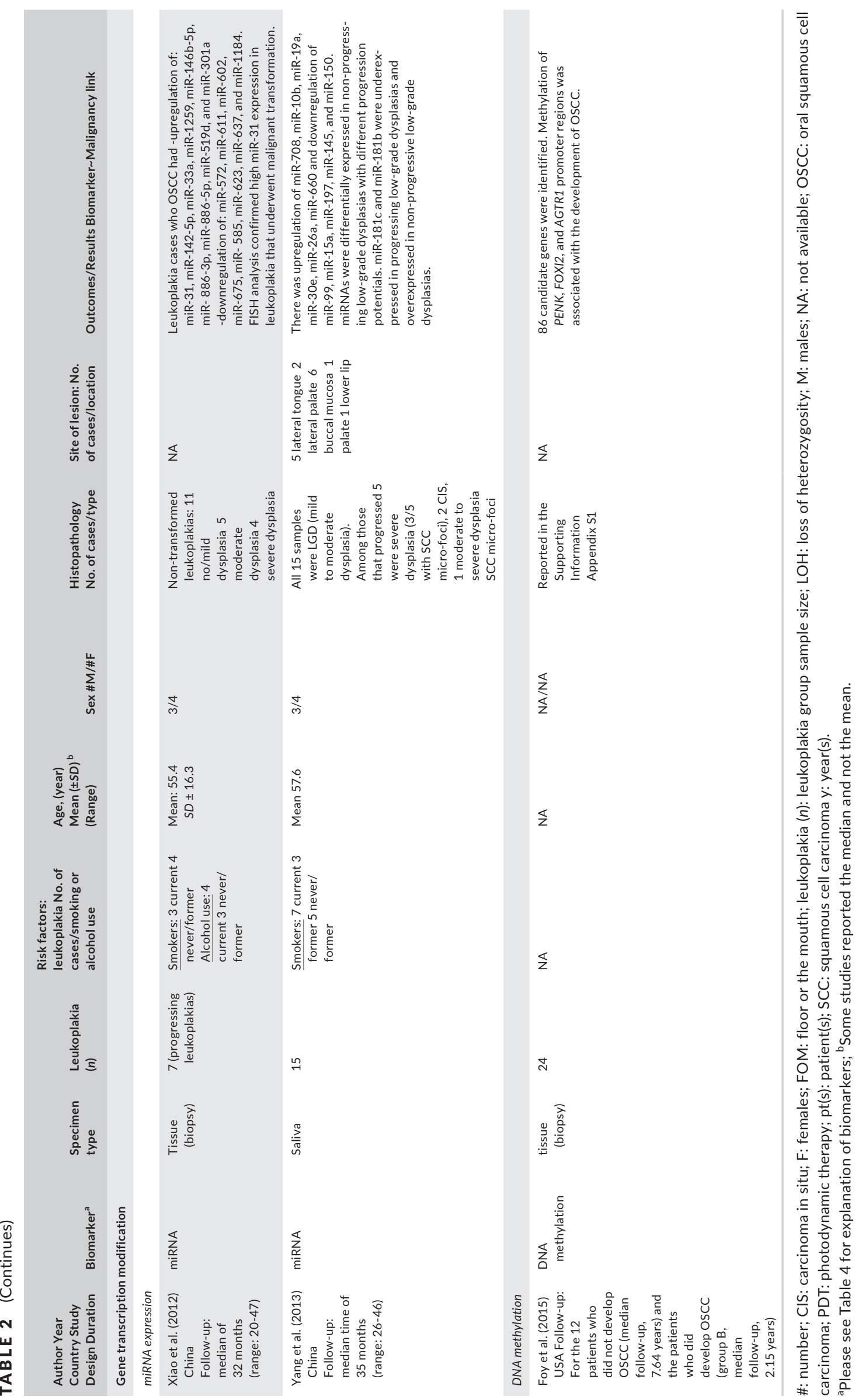


ion channels (Fernandez-Valle, Rodrigo, Garcia-Pedrero, et al., 2016; Fernandez-Valle, Rodrigo, Rodriguez-Santamarta, et al., 2016), sustained angiogenesis factors (Kawaguchi et al., 2008; Lin et al., 2010; Nayak et al., 2015; Saintigny et al., 2009; Yang et al., 2014), and epigenetic biomarkers (Foy et al., 2015; Xiao et al., 2012; Yang et al., 2013). Studies tended to include small sample sizes, under-reported or variably reported histopathological data, did not address potential confounding, reported limited/variable follow-up data, or lacked a control group. Notably, 14 of the 25 studies were components of chemoprevention trials (Beenken et al., 1994; Foy et al., 2015; Kawaguchi et al., 2008; Lee et al., 2000; Mao et al., 1996; Massarelli et al., 2005; Nagao et al., 2017; Rai et al., 2010; Saintigny et al., 2009, 2011, 2018; Uehara et al., 2010; Wan et al., 1999; Yang et al., 2013). Inclusion of subsets from chemoprevention trials may have introduced bias regarding reported malignant transformation rates and accuracy of prognostic biomarkers.

\subsection{Quality assessment of the studies}

Low risk of bias was observed in "statistical reporting," "study participation," "study attrition," and "outcome measurement" (81\%, 70\%, $52 \%$, and $52 \%$ of included studies, respectively), while moderate risk of bias in "study confounding" was found in $70 \%$ of studies and in "outcome measurement," "prognostic factor measurement," and "study attrition" in $48 \%$ of the studies. Percentage of studies with high risk of bias was relatively low and varied between $0 \%$ and $11 \%$ across the different domains (Figure 2; Table 3).

\section{4 | DISCUSSION}

This systematic review was directed to identification of biomarkers that could predict the likelihood of malignant progression over time in patients affected by oral leukoplakia (Table 4). Therefore, only studies applying longitudinal designs were included. Overall, the 25 included studies documented a wide range of biomarkers derived from three different anatomic sources: serum, tissue, and saliva.

\section{Risk of Bias}

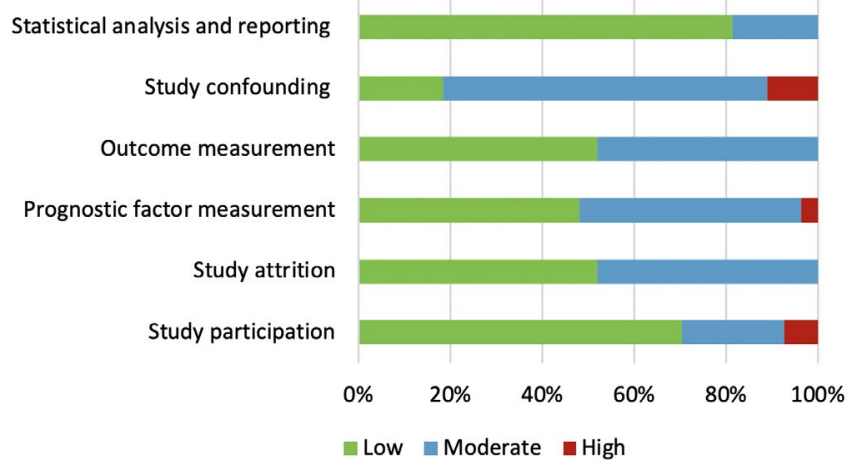

FIGURE 2 Risk of bias in the 25 included studies according to the Quality in Prognosis Studies (QUIPS) criteria (Hayden et al., 2013)
Heterogeneity of biomarkers investigated across these studies precluded direct interstudy comparison in virtually all cases.

Whereas selected biomarkers that were examined longitudinally seem promising, major limitations delineated in the current studies prevent definitive clinical application at this time. Most of the studies: (a) included small sample sizes with the largest comprising only 162 patients (Kawaguchi et al., 2008); (b) suffered from a paucity of histopathological data for leukoplakia and a lack of reporting of demographics and potentially confounding factors such as age, tobacco smoking, and alcohol consumption (Table 2); (c) reported on limited and variable follow-up data and/or did not include a control group. As such, no negative or positive predictive value was reported for the biomarkers. Each study included in the review evaluated distinct biomarkers, with the exception of podoplanin that was evaluated by two research groups (Kawaguchi et al., 2008; Saintigny et al., 2009), but continues to require further investigation. Thus, potential validity of biomarkers examined to date remains to be demonstrated in other studies. Several included papers reported on biomarkers examined in the context of chemoprevention trials (14/25 studies) where intervention may have potentially impacted the malignant transformation rates and accuracy of prognostic biomarkers relative to risk detection for malignant progression of oral leukoplakia (Beenken et al., 1994; Foy et al., 2015; Kawaguchi et al., 2008; Lee et al., 2000; Mao et al., 1996; Massarelli et al., 2005; Nagao et al., 2017; Rai et al., 2010; Saintigny et al., 2009, 2011, 2018; Uehara et al., 2010; Wan et al., 1999; Yang et al., 2013). Finally, most studies exhibited a moderate risk of bias in association with missing data, no consideration of potential confounding, and availability of limited follow-up data (Table 3). Future studies should include a longer follow-up (the longest one reported in the studies included for this systematic review was 7.5 years; by Saintigny et al., 2009) to capture all cases of oral leukoplakia that undergo malignant transformation. This requires a larger effort with multiple centers and resources involved. Notably, biomarker assessment was not conducted in a blinded manner in any of the included studies, further introducing additional potential for bias.

Ten studies aimed to identify objectively measured molecular biomarkers for potential utility in identifying patients with oral leukoplakia at higher risk of malignant transformation. The remaining 14 studies that incorporated biomarker assessment as part of chemoprevention trials had other objectives. We defined the potential role of each of the biomarkers reported in these studies based on their relational juxtaposition to essential hallmarks of cancer (Hanahan \& Weinberg, 2011).

Based on evidence available to date, candidate biomarkers for cancer progression in patients affected by leukoplakia examined in this systematic review lacked substantive evidence as harbingers of risk for malignant transformation of oral leukoplakia. However, we only included those studies that specifically described "leukoplakia"; studies that described dysplasia only were excluded. Consequently, some biomarkers with prognostic value for oral dysplastic lesions (and possibly some leukoplakia cases) may have been missed. In addition, some of these biomarkers were captured in the $\mathrm{Y} 3$, Y4, or Y5 


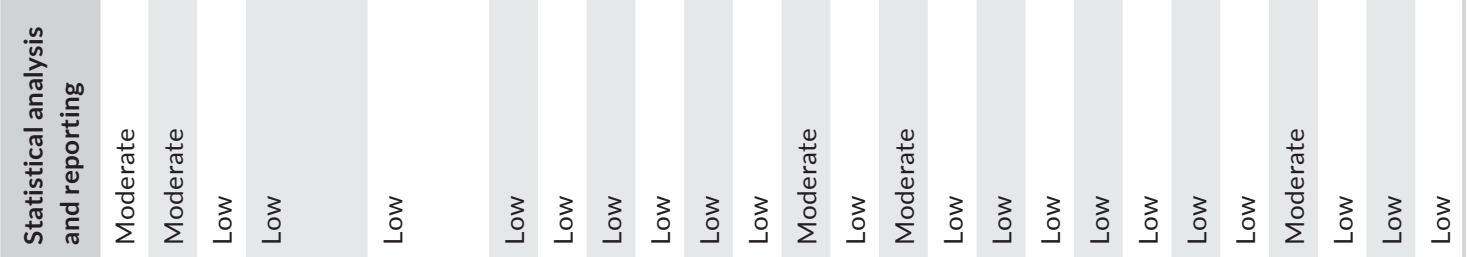

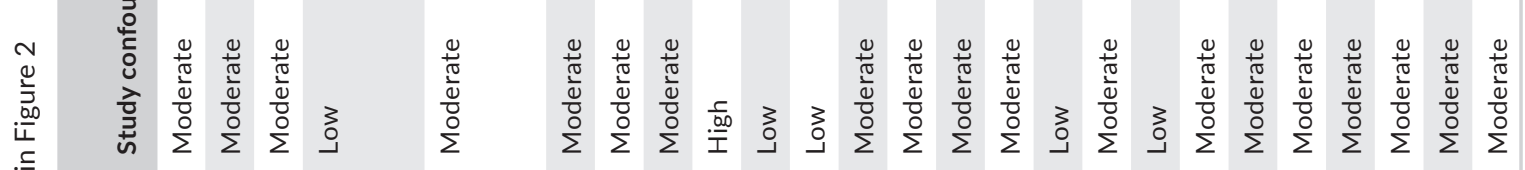



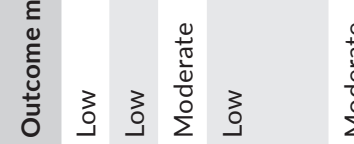

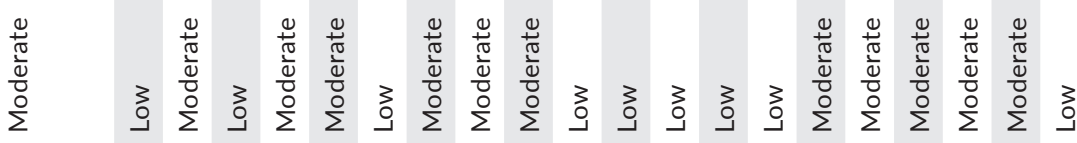

竞

气ั

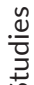

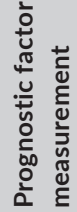

$\stackrel{\frac{\pi}{\pi}}{\frac{\pi}{0}}$

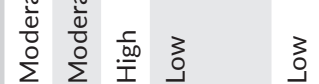

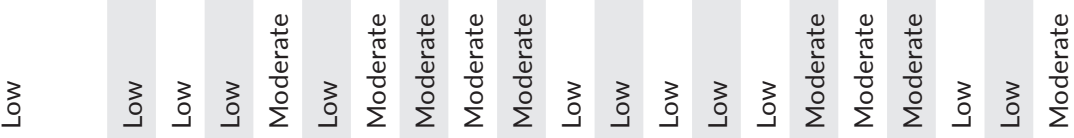

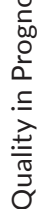

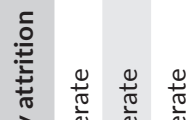

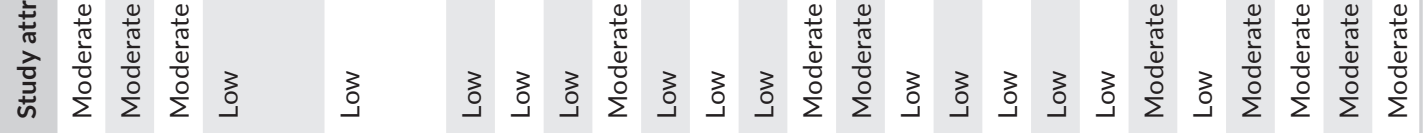

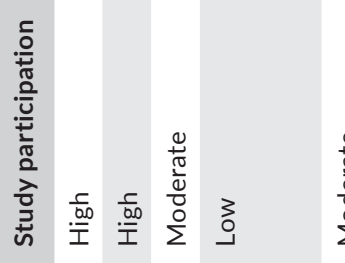

$\frac{\stackrel{0}{\pi}}{\frac{\pi}{0}}$

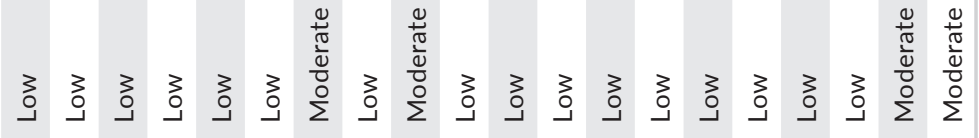

岕

$\frac{\sqrt{3}}{\frac{\pi}{3}}$

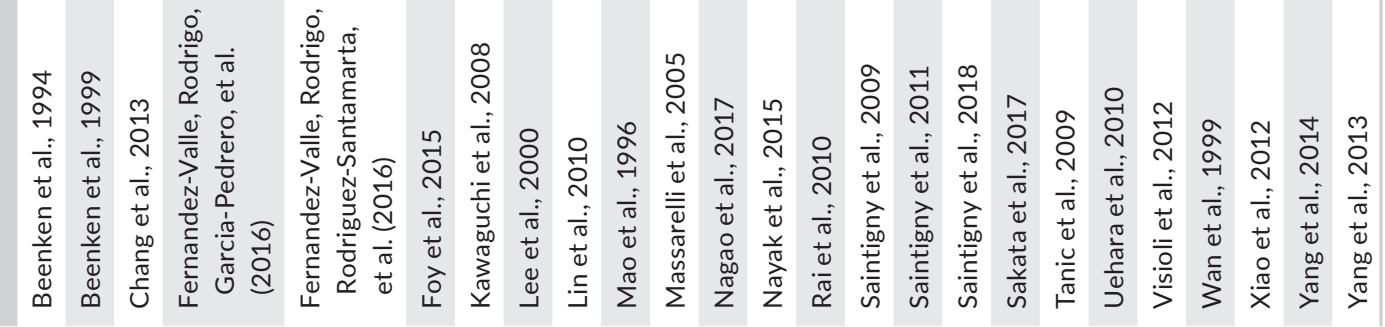


TAB LE 4 Biomarkers used with leukoplakia specimens in the 25 studies displayed in Table 2

\begin{tabular}{|c|c|}
\hline Biomarker acronym & Biomarker name and function \\
\hline $\mathrm{CP}$ & Chromosomal polysomy \\
\hline CRP & C-reactive protein; acute inflammatory marker \\
\hline E-cadherin & Molecule that makes cells adhere to each other (cadherin = "calcium-dependent adhesion") \\
\hline eGFR, EGFR & Epidermal growth factor receptor \\
\hline E-selectin & Cell adhesion molecule only expressed on endothelial cells activated by cytokines; inflammatory marker \\
\hline FGF & Basic fibroblast growth factor and signaling protein \\
\hline FGFR & Fibroblast growth factor receptor \\
\hline GDF & Growth differentiation factor \\
\hline GRP & Glucose-regulated protein \\
\hline HER & Human epidermal growth factor receptor \\
\hline HERG & Human ether-à-go-go related gene \\
\hline ICAM & Intercellular adhesion molecule; inflammatory marker \\
\hline IL & Interleukin, cytokines (secreted proteins, signal molecules) \\
\hline Ki-67 & Antigen, nuclear protein \\
\hline Kv3.4 & Potassium voltage-gated channel gene \\
\hline M-CSF & Macrophage colony-stimulating factor; inflammatory marker \\
\hline MET & Mesenchymal-epithelial transition \\
\hline MDA & Malondialdehyde; oxidative marker \\
\hline miRNA & Micro-ribonucleic acid \\
\hline MMP & Matrix metalloproteinase \\
\hline MYC & Family of regulator genes and proto-oncogenes that code for transcription factors (MyC: Myelocytomatosis) \\
\hline$\Delta \mathrm{Np} 63$ & Tumor protein p63 \\
\hline 8-OHdG & 8-hydroxydeoxyguanosine \\
\hline p21WAF1 & Tumor protein, cell cycle protein regulators \\
\hline p27 & Protein that regulates the cell cycle \\
\hline p53 & Tumor protein, cell cycle protein regulators \\
\hline Podoplanin & Mucin-type protein, specific lymphatic vessel marker \\
\hline RAR- $\beta$ & Retinoic acid receptor-Beta \\
\hline SAA & Serum amyloid $A$; inflammatory marker \\
\hline SMAD4 & Mothers against decapentaplegic homolog 4 gene \\
\hline TGF & Transforming growth factor; inflammatory marker \\
\hline
\end{tabular}

categories and will be reviewed separately in future publications by our group. One example of such a biomarker comes from the study by Zhang and colleagues who examined 296 oral premalignant lesions and showed that LOH at $3 p$ and/or 9p was present in $20 \%$ of cases that underwent malignant transformation (Zhang et al., 2012), further supporting the role of $\mathrm{LOH}$ in malignant transformation of oral leukoplakia. Although $\mathrm{LOH}$ may indeed be a predictive biomarker for malignant transformation, this systematic review highlights that both clinical and histopathological descriptors of lesions should be included in future studies on the topic to ensure robustness of data, but also to allow subsequent meta-analyses should more than one study investigate the same marker or set of markers.

This systematic review disclosed that insufficient longitudinal evidence is currently available to support identification of biomarkers that could improve current methods for detection of leukoplakia and any subsequent malignant disease progression. Therefore, the strength of the current evidence precludes advancement of any candidate biomarkers examined to date into clinical practice. While a variety of technologies and laboratory techniques were employed to measure biomarker expression (including IHC, PCR, ELISA, and next-generation sequencing), outcomes of these studies based on the current body of evidence do not achieve the standards required to identify and validate candidate biomarkers investigated to date. While recent advancements in biomarker development using microarray technology, mass spectrometry, next-generation sequencing, and proteomic technologies have facilitated accomplishment of relatively rapid screening in real time, one of the major challenges that remain unaddressed is the validation in large longitudinal trials to assess the true effectiveness of these biomarkers. Reliable, sensitive, and specific biomarkers are needed to predict malignant transformation in patients affected by leukoplakia and inform physicians on correct treatment decisions. Thus, their relative value 
remains equivocal pending validation in future appropriately designed studies, with no advances currently observed. Well-designed, randomized controlled studies with proper follow-up are required to propose and validate biomarkers for the purpose of establishing a new basis for their clinical and diagnostic utility. Consequently, clinicians continue to rely on tissue biopsy and histopathological assessment of oral dysplasia as the gold standard to determine the risk of malignant transformation and management strategies for patients with oral leukoplakia.

Limitations in tissue availability of leukoplakia samples (often small, formalin-fixed paraffin-embedded) and the preinvasive nature of leukoplakia may limit its systemic detectable expression in saliva and blood. Two promising biomarkers identified in this systematic review included podoplanin and cell cycle regulators. Podoplanin was identified as a possible independent predictor for cancer progression (hazard ratio $=3.1 ; 95 \% \mathrm{Cl}$ : 1.5-6.2) (Kawaguchi et al., 2008), and p27 loss was shown to be an independent factor for malignant transformation ( $p=0.02$ ) (Massarelli et al., 2005). However, despite the potential for greatly improving current clinical tools and collective recent advances, there is still insufficient longitudinal evidence to support the identification of these and other biomarkers that could improve the current methods for detection of leukoplakia and any subsequent malignant disease progression based on the 25 studies that met eligibility for inclusion in this systematic review. Thus, oral epithelial dysplasia on histopathological assessment of a biopsy specimen currently remains the best predictor for transformation to invasive squamous cell carcinoma.

\subsection{Future directions}

The past decade has seen an increase in availability and access to high-throughput profiling and sequencing technologies for genomic analysis and increased bioinformatic approaches to support rapid and comprehensive investigations of multi-omics data in the precancer and cancer research domain. The growing capacity for integration and systems analysis of multi-omics data needs to be pursued to help elucidate interactions between genetic and epigenetic alterations being recognized in leukoplakia and promote identification of a prognostic biomarker or panel of biomarkers with improved potential for cancer prediction and simultaneously definition of pathways involved in malignant transformation including candidate molecules which could be targeted by therapeutic interventions to impede progression of oral leukoplakia to invasive cancer. Notably, the era of precision medicine has seen achievement of this goal in the context of some cancers, including breast cancer. Achievement of this goal is further linked to definition of specific genetic alterations associated with leukoplakia and processes that drive malignant transformation. Increased understanding of these processes will also promote further identification of candidate biomarkers that may represent detectable molecular signals arising as a consequence of transformational processes. In the future, additional well-designed research that defines triggers of leukoplakia progression through integrated, ad hoc computational analyses is needed to leverage genomic and other meta-data in defining appropriate candidate biomarkers and tailored molecular targets with translational potential to mitigate risk of malignant transformation associated with leukoplakia.

As more high-throughput technologies such as next-generation sequencing are utilized in the discovery of diagnostic, prognostic, and predictive biomarkers, it is incumbent on researchers to better understand biomarker types and to better plan study designs to suit the biomarker type but also the question at hand. In the case of oral leukoplakia malignant transformation, assessing DNA tissue biomarkers through genome-wide association studies or even exome sequencing in affected individuals may provide insight into genetic mutations or aberrations of susceptibility in affected individuals, and, if followed up over time, may lead to discovery of a susceptibility profile for malignant transformation, as it is known that not all leukoplakias progress to malignancy. These biomarkers can be tested retrospectively on biobanked samples, and would hasten biomarker discovery as patient recruitment for biomarker study design would not necessarily need to proceed prospectively. DNA tumor biomarkers of oral leukoplakia samples themselves may offer equally interesting insights, but would require a prospective study design. These biomarkers would be amenable for testing therapy response and pharmacodynamics, but may suffer from lack of reproducibility as different somatic mutations may be present in different parts of the lesion, thus highlighting the importance of biopsy site selection and multiple sampling of oral leukoplakia. Nonetheless, having this insight would dramatically increase our ability to discuss treatment options with patients, and would be invaluable prognostic predictors for therapy. Finally, RNA, protein, or metabolite biomarkers can be assayed as DNA tumor biomarkers, but have the advantage of being tested in a variety of biosamples including blood, saliva, gingival crevicular fluid, tissue, or cell scrapes, would equally be amenable to therapy testing and monitoring of response, would provide reproducibility at any one point in time, and could be used in prospective study designs, but additionally would be suitable as surrogate markers of disease. Furthermore, their discovery can elucidate molecular pathways involved in malignant transformation and provide more comprehensive insights into this process.

Ideally, collaborative research, at the international level, strategically designed to systematically collect and analyze data is required in order to (a) achieve a robust, adequately powered study population for follow-up of patients with leukoplakia and (b) observe progression in a larger subset of patients than is normally possible in single-center studies due to the relatively low rate of malignant transformation. Patients with newly diagnosed leukoplakia should be included with subsequent longitudinal follow-up, and collection of structured data at specifically defined time points. Future studies should also focus on identifying biomarkers by integrating -omics data with environmental and lifestyle variables with the final goal of identifying more informative predictors of cancer progression that supersede dependence on histopathological diagnosis alone. Collection of these data into a centralized repository in the context of good histopathology data would further create a resource amenable to application of bioinformatic approaches and predictive 
modeling to identify the most informative variables for screening across time.

\section{5 | CONCLUSIONS}

This review identified insufficient longitudinal evidence to support validated prognostic biomarkers for oral leukoplakia. Further studies are needed to identify molecular targets with the potential to mitigate risk of malignant transformation of oral leukoplakia to advance precision medicine approaches to management of patients with these lesions.

\section{ACKNOWLEDGEMENTS}

The WWOM VII Steering Committee gratefully acknowledges the following organizations and companies that provided financial support for WWOM VII: American Academy of Oral Medicine, European Association of Oral Medicine, The British Society for Oral Medicine, Oral Diseases, Henry Schein Cares Foundation, Colgate Palmolive, Xerostom, Afyx, The World Dental Education Foundation, and Unilever. In addition, research reported in this publication was supported by the National Institute Of Dental \& Craniofacial Research of the National Institutes of Health under Award Number R13DE027613. The content is solely the responsibility of the authors and does not necessarily represent the official views of the National Institutes of Health.

\section{CONFLICT OF INTEREST}

The authors have no conflict of interest. The authors would like to thank Dr. Jim Berryman, Liaison Librarian, Brownless Biomedical Library, University Library, The University of Melbourne, for his guidance developing the literature search strategy.

\section{AUTHOR CONTRIBUTION}

Alessandro Villa conceptualization, data collection, paper writing. Antonio Celentano conceptualization, data collection, critical review of the paper. Ingrid Glurich conceptualization, critical review of the paper. Wenche S. Borgnakke conceptualization, critical review of the paper. Siri Beier Jensen critical review of the paper. Douglas E. Peterson critical review of the paper. Konstantina Dellicritical review of the paper. David Ojedacritical review of the paper. Arjan Vissink critical review of the paper. Camile S. Farah conceptualization, critical review of the paper.

\section{ORCID}

Alessandro Villa (iD https://orcid.org/0000-0002-1966-6000

Douglas E. Peterson (iD https://orcid.org/0000-0002-2665-4964

Konstantina Delli iD https://orcid.org/0000-0003-3115-3977
Arjan Vissink (iD https://orcid.org/0000-0003-2581-4361

Camile S. Farah (iD https://orcid.org/0000-0002-1642-6204

\section{REFERENCES}

Beenken, S. W., Huang, P., Sellers, M., Peters, G., Listinsky, C., Stockard, C., ... Grizzle, W. (1994). Retinoid modulation of biomarkers in oral leukoplakia/dysplasia. Journal of Cellular Biochemistry Supplement, 19, 270-277.

Beenken,S.W.,Sellers, M.T.,Huang,P.,Peters, G., Krontiras, H., Dixon, P., ... Grizzle, W. E. (1999). Transforming growth factor alpha (tgf-alpha) expression in dysplastic oral leukoplakia: modulation by 13-cis retinoic acid. Head and Neck, 21, 566-573. https://doi.org/10.1002/ (SICI)1097-0347(199909)21:6<566:AID-HED11>3.0.CO;2-H

Bray, F., Ferlay, J., Soerjomataram, I., Siegel, R. L., Torre, L. A., \& Jemal, A. (2018). Global cancer statistics 2018: Globocan estimates of incidence and mortality worldwide for 36 cancers in 185 countries. CA: A Cancer Journal for Clinicians, 68, 394-424. https://doi.org/10.3322/caac.21492

Chang, P. Y., Kuo, Y. B., Wu, T. L., Liao, C. T., Sun, Y. C., Yen, T. C., \& Chan, E. C. (2013). Association and prognostic value of serum inflammation markers in patients with leukoplakia and oral cavity cancer. Clinical Chemistry and Laboratory Medicine, 51, 1291-1300. https:// doi.org/10.1515/cclm-2012-0504

D'Amico, M., Gasparoli, L., \& Arcangeli, A. (2013). Potassium channels: Novel emerging biomarkers and targets for therapy in cancer. Recent Patents on Anti-cancer Drug Discovery, 8(1), 53-65. https://doi. org/10.2174/15748928130106

Fernandez-Valle, A., Rodrigo, J. P., Garcia-Pedrero, J. M., RodriguezSantamarta, T., Allonca, E., Lequerica-Fernandez, P., \& de Vicente, J. C. (2016). Expression of the voltage-gated potassium channel kv3.4 in oral leucoplakias and oral squamous cell carcinomas. Histopathology, 69, 91-98. https://doi.org/10.1111/his.12917

Fernandez-Valle, A., Rodrigo, J. P., Rodriguez-Santamarta, T., Villaronga, M. A., Alvarez-Teijeiro, S., Garcia-Pedrero, J. M., ... de Vicente, J. C. (2016). HERG1 potassium channel expression in potentially malignant disorders of the oral mucosa and prognostic relevance in oral squamous cell carcinoma. Head and Neck, 38, 1672-1678. https://doi. org/10.1002/hed.24493

Foy, J. P., Pickering, C. R., Papadimitrakopoulou, V. A., Jelinek, J., Lin, S. H., William, W. N. Jr, ... Saintigny, P. (2015). New DNA methylation markers and global DNA hypomethylation are associated with oral cancer development. Cancer Prevention Research, 8, 1027-1035. https://doi.org/10.1158/1940-6207.CAPR-14-0179

Hanahan, D., \& Weinberg, R. A. (2011). Hallmarks of cancer: The next generation. Cell, 144(5), 646-674. https://doi.org/10.1016/j. cell.2011.02.013

Hayden, J. A., van der Windt, D. A., Cartwright, J. L., Cote, P., \& Bombardier, C. (2013). Assessing bias in studies of prognostic factors. Annals of Internal Medicine, 158, 280-286. https://doi. org/10.7326/0003-4819-158-4-201302190-00009

Kawaguchi, H., El-Naggar, A. K., Papadimitrakopoulou, V., Ren, H., Fan, Y. H., Feng, L., ... Mao, L. (2008). Podoplanin: A novel marker for oral cancer risk in patients with oral premalignancy. Journal of Clinical Oncology, 26, 354-360. https://doi.org/10.1200/JCO.2007.13.4072

Lee, J. J., Hong, W. K., Hittelman, W. N., Mao, L., Lotan, R., Shin, D. M., ... Lippman, S. M. (2000). Predicting cancer development in oral leukoplakia: Ten years of translational research. Clinical Cancer Research, 6, 1702-1710.

Lin, C. Y., Chen, W. H., Liao, C. T., Chen, I. H., Chiu, C. C., Wang, H. M., ... Cheng, A. J. (2010). Positive association of glucose-regulated protein 78 during oral cancer progression and the prognostic value in oral precancerous lesions. Head and Neck, 32, 1028-1039. https://doi. org/10.1002/hed.21287 
Mao, L., Lee, J. S., Fan, Y. H., Ro, J. Y., Batsakis, J. G., Lippman, S., ... Hong, W. K. (1996). Frequent microsatellite alterations at chromosomes 9 p21 and 3p14 in oral premalignant lesions and their value in cancer risk assessment. Nature Medicine, 2, 682-685.

Massarelli, E., Brown, E., Tran, N. K., Liu, D. D., Izzo, J. G., Lee, J. J., ... Papadimitrakopoulou, V. A. (2005). Loss of E-cadherin and p27 expression is associated with head and neck squamous tumorigenesis. Cancer, 103, 952-959. https://doi.org/10.1002/cncr.20879

Mello, F. W., Miguel, A. F. P., Dutra, K. L., Porporatti, A. L., Warnakulasuriya, S., Guerra, E. N. S., \& Rivero, E. R. C. (2018). Prevalence of oral potentially malignant disorders: A systematic review and meta-analysis. Journal of Oral Pathology \& Medicine, 47, 633-640. https://doi. org/10.1111/jop.12726

Moher, D., Liberati, A., Tetzlaff, J., \& Altman, D. G. (2009). Preferred reporting items for systematic reviews and meta-analyses: The PRISMA statement. PLoS Medicine, 6(7), e1000097. https://doi.org/10.1371/ journal.pmed.1000097

Nagao, T., Warnakulasuriya, S., Sakuma, H., Miyabe, S., Hasegawa, S., Machida, J., ... Hashimoto, S. (2017). P53 and ki67 as biomarkers in determining response to chemoprevention for oral leukoplakia. Journal of Oral Pathology \& Medicine, 46, 346-352. https://doi. org/10.1111/jop.12498

Napier, S. S., \& Speight, P. M. (2008). Natural history of potentially malignant oral lesions and conditions: An overview of the literature. Journal of Oral Pathology \& Medicine, 37, 1-10. https://doi. org/10.1111/j.1600-0714.2007.00579.x

Nayak, S., Goel, M. M., Makker, A., Bhatia, V., Chandra, S., Kumar, S., \& Agarwal, S. P. (2015). Fibroblast growth factor (FGF-2) and its receptors FGFR-2 and FGFR-3 may be putative biomarkers of malignant transformation of potentially malignant oral lesions into oral squamous cell carcinoma. PLoS ONE, 10, e0138801. https://doi. org/10.1371/journal.pone.0138801

Rai, B., Kaur, J., Jacobs, R., \& Singh, J. (2010). Possible action mechanism for curcumin in pre-cancerous lesions based on serum and salivary markers of oxidative stress. Journal of Oral Science, 52, 251-256. https://doi.org/10.2334/josnusd.52.251

Reibel, J. (2003). Prognosis of oral pre-malignant lesions: Significance of clinical, histopathological, and molecular biological characteristics. Critical Reviews in Oral Biology and Medicine, 14, 47-62. https://doi. org/10.1177/154411130301400105

Saintigny, P., El-Naggar, A. K., Papadimitrakopoulou, V., Ren, H., Fan, Y. H., Feng, L., ... Mao, L. (2009). Deltanp63 overexpression, alone and in combination with other biomarkers, predicts the development of oral cancer in patients with leukoplakia. Clinical Cancer Research, 15, 6284-6291. https://doi.org/10.1158/1078-0432.CCR-09-0498

Saintigny, P., William, W. N. Jr, Foy, J. P., Papadimitrakopoulou, V., Lang, W., Zhang, L., ... Lippman, S. M. (2018). Met receptor tyrosine kinase and chemoprevention of oral cancer. Journal of the National Cancer Institute, 110, 01. https://doi.org/10.1093/jnci/djx186

Saintigny, P., Zhang, L., Fan, Y. H., El-Naggar, A. K., Papadimitrakopoulou, V. A., Feng, L., ... Mao, L. (2011). Gene expression profiling predicts the development of oral cancer. Cancer Prevention Research, 4, 218229. https://doi.org/10.1158/1940-6207.CAPR-10-0155

Sakata, J., Yoshida, R., Matsuoka, Y., Nagata, M., Hirosue, A., Kawahara, K., ... Nakayama, H. (2017). Predictive value of the combination of SMAD4 expression and lymphocyte infiltration in malignant transformation of oral leukoplakia. Cancer Medicine, 6, 730-738. https:// doi.org/10.1002/cam4.1005

Speight, P. M., Khurram, S. A., \& Kujan, O. (2018). Oral potentially malignant disorders: Risk of progression to malignancy. Oral Surgery, Oral Medicine, Oral Pathology and Oral Radiology, 125, 612-627. https:// doi.org/10.1016/j.0ooo.2017.12.011
Tanic, N., Tanic, N., Milasin, J., Vukadinovic, M., \& Dimitrijevic, B. (2009). Genomic instability and tumor-specific DNA alterations in oral leukoplakias. European Journal of Oral Sciences, 117, 231-237. https:// doi.org/10.1111/j.1600-0722.2009.00624.x

Uehara, M., Ikeda, H., Nonaka, M., \& Asahina, I. (2010). Histopathological change of oral malignant tumour and epithelial dysplasia subjected to photodynamic therapy. Journal of Oral \& Maxillofacial Research, 1, e5. https://doi.org/10.5037/jomr.2010.1305

Villa, A., \& Woo, S. B. (2017). Leukoplakia - a diagnostic and management algorithm. Journal of Oral and Maxillofacial Surgery, 75, 723-734. https://doi.org/10.1016/j.joms.2016.10.012

Visioli, F., Lauxen, I. S., Sant'ana Filho, M., \& Rados, P. V. (2012). Expression of the cell cycle regulation proteins p53 and p21WAF1 in different types of non-dysplastic leukoplakias. Journal of Applied Oral Science, 20, 369-375. https://doi.org/10.1590/S1678-77572012000300013

van der Waal, I., \& Axell, T. (2002). Oral leukoplakia: A proposal for uniform reporting. Oral Oncology, 38, 521-526. https://doi.org/10.1016/ S1368-8375(01)00125-7

Wan, X. S., Meyskens, F. L. Jr, Armstrong, W. B., Taylor, T. H., \& Kennedy, A. R. (1999). Relationship between protease activity and neu oncogene expression in patients with oral leukoplakia treated with the Bowman Birk inhibitor. Cancer Epidemiology, Biomarkers \& Prevention, 8, 601-608.

Warnakulasuriya, S. (2018). Clinical features and presentation of oral potentially malignant disorders. Oral Surgery, Oral Medicine, Oral Pathology and Oral Radiology, 125, 582-590. https://doi.org/10.1016/j.00oo.2018.03.011

Warnakulasuriya, S., Johnson, N. W., \& van der Waal, I. (2007). Nomenclature and classification of potentially malignant disorders of the oral mucosa. Journal of Oral Pathology \& Medicine, 36, 575-580. https://doi.org/10.1111/j.1600-0714.2007.00582.x

Xiao, W., Bao, Z. X., Zhang, C. Y., Zhang, X. Y., Shi, L. J., Zhou, Z. T., \& Jiang, W. W. (2012). Upregulation of miR-31 is negatively associated with recurrent/newly formed oral leukoplakia. PLOS ONE, 7, e0038648. https://doi.org/10.1371/journal.pone.0038648

Yang, Y., Li, Y. X., Yang, X., Jiang, L., Zhou, Z. J., \& Zhu, Y. Q. (2013). Progress risk assessment of oral premalignant lesions with saliva miRNA analysis. BMC Cancer, 13, 129. https://doi.org/10.1186/1471-2407-13-129

Yang, C. Z., Ma, J., Luo, Q. Q., Neskey, D. M., Zhu, D. W., Liu, Y., ... Zhong, L. P. (2014). Elevated level of serum growth differentiation factor 15 is associated with oral leukoplakia and oral squamous cell carcinoma. Journal of Oral Pathology \& Medicine, 43, 28-34. https://doi. org/10.1111/jop.12091

Zhang, L., Poh, C. F., Williams, M., Laronde, D. M., Berean, K., Gardner, P. J., \& Rosin, M. P. (2012). Loss of Heterozygosity (LOH) profiles Validated risk predictors for progression to oral. Cancer Prevention Research (Philadelphia PA), 5, 1081-9. https://doi.org/10.1158/19406207.CAPR-12-0173

\section{SUPPORTING INFORMATION}

Additional supporting information may be found online in the Supporting Information section at the end of the article.

How to cite this article: Villa A, Celentano A, Glurich I, et al. World Workshop on Oral Medicine VII: Prognostic biomarkers in oral leukoplakia: A systematic review of longitudinal studies. Oral Dis. 2019;25(Suppl. 1):64-78. https://doi.org/10.1111/ odi.13087 